\title{
Research on Coal Seam Spontaneous Combustion Mutation and Precise Detection Technology
}

\author{
Zhongping Cui1,a, *Xianfeng Liu2, 3,b, Jinxin Huang2,3,c, Jiabin \\ Zhang2,3,d , Yangyang Meng2,3,e \\ 1Jinzhong Coal Planning and Design Institution,Yuci, Shanxi \\ 030600,P.R. China; \\ 2School of Resource and Safety Engineering, China University of Ming \& \\ Technology(Beijing),Beijing 100083, P.R. China; \\ 3State Key Laboratory of Coal Resource and Safe Ming, Beijing 100083, \\ P.R. China; \\ aemail:460637881@qq.com, bemail:cumtblxf@163.com,cemail: \\ 846150793@qq.com, \\ demail: 422781965@qq.com, eemail: 1091421385@qq.com
}

\begin{abstract}
The cusp catastrophe theory was used to establish the mine spontaneous combustion mutation model. Factors of spontaneous combustion were processed in quantization. Important conclusions were obtained about the mine combustion prevention and control.The application effect of cusp catastrophe theory and precise detection technology of coal seam spontaneous combustion location applied in Ximing Coal Mine was introduced. Practice has proved that this technology is the key to solving the problems of coal seam spontaneous combustion prevention. It has great promotional value.
\end{abstract}

Keywords: Spontaneous Combustion; Cusp Catastrophe; Mutation Model; Isotopic Radon Measurement Method; Fire Source Detection

\section{Introduction}

Mine fire is one of the major disasters underground coal mine in China, which is a serious threat to the coal mine's safety production. $47 \%$ of state-owned coal mines have the tendency of spontaneous combustion. According to statistics, the direct loss of coal resources is up to 10-million tons every year because of the coal spontaneous combustion, and 200-million tons of high quality coal are lost indirectly. The mine fire not only damages a lot of coal resources, but also leads to a series of issues on geological environment, ecological environment, and atmospheric environment[1]. Therefore, as the important research content of mine fire hazard source identification, spontaneous combustion prevention and control is an urgent task. Scholars at home and 
abroad have studied it from the nonlinear perspective, but so far the research is still not profound enough. Based on the present situation, this article starts from the branch theory of nonlinear(catastrophe theory).

Catastrophe theory has been used to deeply analysis the development mechanism of this nonlinear process - coal spontaneous combustion. Isotopic radon measurement method is used to accurately detect the position of the source of coal seam spontaneous combustion fire[2]. Various internal and external factors that influence the mine spontaneous fire have been summarized. According to the knowledge of two kinds of hazard source theory and catastrophe theory, mine spontaneous fire mutation model is established, and the examples is analyzed. Some useful conclusions have been obtained to improve coal mine's safety production in our country, especially useful to control the spontaneous combustion fire.

\section{Catastrophe theory}

1.1 Introduction to the theory of mutation

Catastrophe theory is an emerging mathematical branch subject which studies discontinuity and singular point. It was founded by the French mathematician Rene Tom in 1972. Catastrophe theory was developed on the basis of modern mathematics like collection, topology, differential geometry, group theory, manifold and so on[3]. It has two kinds of description form, one is direct described in the form of modern mathematics called higher mutation theory; the other one which building on the foundation of modern mathematics thought, but using the tools borrowed from classical analysis mathematics to describe, is called the basic theory of mutation[4]. Higher mutation theory is very profound, and the basic theory of mutation is enough to solve general application problems. Catastrophe theory is a developing discipline. It develops while combining and crossing with other discipline in a wide range of application process. Its development and application prospects are very wide.

1.2 Foundation of mutation theory

Catastrophe theory originated in the Hassler Whitney's singularity of smooth mapping and Poincare and Andrianof's bifurcation theory of dynamical system. The main theoretical basis are singularity theory, critical point theory, structural stability, splitting lemma and codimension. This theory includes seven elementary mutations: folding mutation[5], sharp point mutations, dovetail umbilical point mutation[6], elliptic type umbilic, hyperbolic umbilical point, butterfly mutation[7], parabolic point. As an emerging discipline, catastrophe theory has been widely used in various research areas , such as coal mine fire, coal and rock instability, mechanism, transportation, machinery, etc[8-14].

\section{Quantitative processing of the factors that affect coal spontaneous combustion}

The two types of hazard theory shows that in the process of coal spontaneous combustion, the first category of hazards $U$ is the internal factor set of coal 
spontaneous combustion that have influence of spontaneous combustion tendency, the second category of hazards $\mathrm{V}$ is the external factors set affecting coal spontaneous combustion[15]. The spontaneous combustion tendency of coal is the main influencing factors of coal spontaneous combustion fire accident, and geological conditions, development conditions, mining conditions, ventilation conditions these external factors determine the possibility of spontaneous fire accidents.

The model reflecting the first category of hazard U' relationship with other related factors accurately can be described like the formula below:

$$
u=f_{u}\left(u_{1}, u_{2}, \ldots, u_{m}\right)
$$

Where, $\mathrm{u}$ is the number of the first kind of hazards $\mathrm{U}$; $\mathrm{Ui}$ is the gas proportion of $\mathrm{i}$ occurring in the process of coal spontaneous combustion. For example, carbon monoxide, carbon dioxide and moisture, volatile matter, sulfur dioxide, hydrogen sulphide, ethane, propane, ethylene, propylene, benzene, etc.

The model reflecting the second category of hazard V' relationship with other related factors accurately can be described like the formula below:

$$
v=f_{v}\left(v_{1}, v_{2}, \ldots, v_{n}\right)
$$

Where, $\mathrm{v} 1$ is the number of the first kind of hazards $\mathrm{V}$; Vi is external factors affecting coal spontaneous combustion: concentration of air, oxygen concentration, working face angle, length of working face, working face impelling speed, the buried depth of coal seam, mining methods, etc. $(1 \leq \mathrm{i} \leq \mathrm{n})$

Coal spontaneous combustion proceed under the combined action of influencing factors set $\mathrm{U}$ and $\mathrm{V}$. In the process of mine production, external influence factors change largely in different coal seam or even the same coal seam in different periods. As for specific coal seam or mining area, the inner factors set $U$ is relative stable. Development degree of coal spontaneous combustion is presented by variable $\mathrm{X}, \mathrm{X}$ reflects the progress condition of coal spontaneous combustion process and the threat degree of spontaneous combustion to underground workers.

2.2 Mutation model of spontaneous combustion

In this paper, seven kinds of typical elementary mutations-cusp catastrophe are used to analysis the system. According to cusp mutation manifold's most common form of expression and the construction of control variables and state variables in natural mine fire system, a mutation model of spontaneous combustion of manifold expressions has been built :

$$
\left(X-X_{0}\right)^{3}+d u\left(X-X_{0}\right)+v=0
$$

Where, $\mathrm{d}$ and $\mathrm{X} 0$ are undetermined coefficients.

If control the variables $\mathrm{u}, \mathrm{v}$, and each related factor take the linear relationship, namely

$$
u=a_{1} u_{1}+a_{2} u_{2}+\cdots+a_{m} u_{m}+a_{m+1}
$$


(4)

$$
v=b_{1} v_{1}+b_{2} v_{2}+\cdots+b_{m} v_{n}+b_{n+1}
$$

(5)

Where, $\mathrm{a}_{\mathrm{i}}(1 \leq \mathrm{i} \leq \mathrm{m}+1)$ and $\mathrm{b}_{\mathrm{j}}(1 \leq \mathrm{j} \leq \mathrm{n}+1)$ are undetermined coefficients. Plug formula(4) and formula(5) into formula(3), we can get:

$$
\begin{gathered}
\left(X-X_{0}\right)^{3}+d\left(a_{1} u_{1}+a_{2} u_{2}+\cdots+a_{m} u_{m}+\right. \\
\left.a_{m+1}\right)\left(X-X_{0}\right)+b_{1} v_{1}+b_{2} v_{2}+\cdots+b_{n} v_{n}+b_{n+1}=0
\end{gathered}
$$

(3) mutation of manifold is shown in Fig1.

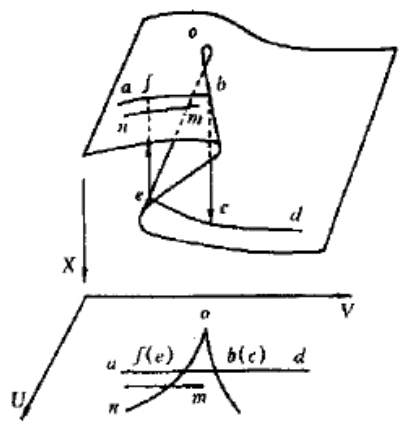

Fig1 Formula(3)'s schematic diagram of catastrophe manifold

2.3 Analysis of spontaneous combustion catastrophe model

As we can see from Fig1, as for a specific coal seam, the value of the controlled variable $u$ is always certain and the variation is small. But as the factors such as advancing of mining working face and goaf ventilation changes constantly, the value of the controlled variable $\mathrm{v}$ also changes constantly. Under the combined effect of both controlled variable $u$ and $v$, mine disasters process of coal spontaneous combustion reflected in the catastrophe manifold is abcd. The whole ab represents coal's low temperature oxidation period and self heating period. In the period of coal's low temperature oxidation period, oxidation process is smooth and slow, the weight of coal increases slightly, chemical activity enhances, oxidation have been activated. The heat produced in the process of low temperature oxidation form initial fluctuations in the system, and release through the crack into the surrounding coal body and environment (one of self-organization evolution mechanism). If external factors still don't get appropriate control, it will meet the self heating period in the process of coal spontaneous combustion. At this stage, the coal oxidation accelerated itself, a lot of heat is released from oxidation exothermic reaction. The heat not only heat surrounding coal body and accelerate the oxidation of exothermic reaction, there will also be some heat feedback back to itself, which accelerate oxidation exothermic reaction itself (one of autoacceleration, autocatalysis and self-organization evolution mechanism). It can be seen that manifold mutations from $\mathrm{a}$ to $\mathrm{b}$ in the figure is a gradual process. When the coal spontaneous 
combustion process reaches state $\mathrm{Xb}$, if continue to develop towards bad direction, the state variable $\mathrm{X}$ will decline from $\mathrm{Xb}$ to Xc. At this time, coal spontaneous combustion process mutates, which leads to coal's spontaneous combustion. In this process, the heat produced by oxidation reaction can't be sent out timely, which make the heat accumulated in the coal body bigger and bigger. When it exceed coal's ignition temperature, the coal will have combustion reaction. Lacked oxygen in coal body, the internal of the coal is smoldering at this time. As smoldering continues, the heat wind in coal body will increases further , pushing air leakage increase further. In the process of thermal fluid migration, the oxidation of exothermic reaction of affected coal continued to accelerate and eventually make the smoldering fire into flame combustion (mutation mechanism) due to having enough oxygen.

The whole process above reflected in the bifurcation set is the process of the abcd passing over the right border of bifurcation set. According to the knowledge of catastrophe theory, we can conclude that the singularity set $\mathrm{S}$ is a subset of the sharp point mutation manifold $M$ that satisfies the equation

$$
\left(X-X_{0}\right)^{3}+d u\left(X-X_{0}\right)+v=0
$$

(7)

and the equation

$$
\left(X-X_{0}\right)^{2}+d u=0
$$

Through eliminating ( $\mathrm{X}-\mathrm{X} 0$ ), we can get from simultaneous equation(3) and (8) that curve $\mathrm{D}$ is decided by the parameters plane equation
4
$d^{3} u^{3}+$
27
$v^{2}=0$

(9)

Curve D is known as the bifurcation set.

Therefore, a necessary condition for coal spontaneous combustion is that the process of coal spontaneous combustion exceeded the boundary of bifurcation set. that is to say it must satisfy the equation(9) :

So, to prevent the coal spontaneous combustion is to prevent the process of exceeded the boundary of bifurcation set. The methods being used currently mainly include voltage sharing,air leakage prevention, grouting, nitrogen injection, etc.

\section{Accurate detection technology of the location of coal seam spontaneous combustion fire}

3.1 Detection principle

There are three natural radioactive series widely in nature, namely, uranium series, thorium series and actinium series. Because of its long half-life, they can be used as the mother nuclide existed in rock, soil, coal and other mediums. Through a series of decay, uranium, thorium and actinium all create radionuclide radon(Rn) which in the form of aerosol state under atmospheric pressure. 
Because of having the longest half-life, radon is often used as information to the study geodynamic phenomena or radon radiation hazards (mainly refers to the decay daughter Rn - 222 of uranium series and its own decay daughter). Radon is radioactive isotope and its decay daughter are solid particles. So the measurement of both the radon and radon's decay daughter can reflect the status of the mother nuclide's form and varying condition. Radon daughter has very strong adsorption capacity, they can firmly adhere to the surface of objects. Therefore varied methods can be used to collect and measure radon and its daughter.

Radon gas migrate upward from underground. The process is vertical movement mainly by convection. When the temperature and pressure condition underground changes, high temperature and pressure produced by spontaneous combustion of coal seam will increase the rate of the migration of radon gas. In this way, radon density at the top of the fire area will be higher than the fire area's radon density before burning. In a word, when other coal seam have heat source while other conditions are similar, the rate and number of radon gas migration will increase greatly. This will produce certain radon concentration differences over the hot area. So the method of measuring radon can be used to describes the change of radon concentration indirectly, in order to accurately determine the position and approximate scope of fire area .

3.2 Detecting method

3.2.1 Working method

With the continuous development of nuclear electronic technology, the methods of radon measurement also improve continuously. Conventional method is let the gas sample enter the detector and then measure it. The ground radon measurement method mainly adopts the strength of alpha rays produced by the measuring radon's decay daughter. According to the detection principle, it can be divided into the alpha cup method, alpha card method, activated carbon method, thermoluminescence method, the measuring method of polonium, track etching method, etc. According to the detection technology, it can be divided into the instantaneous radon measurement method and cumulative radon measurement method. According to different conditions and detection accuracy, all the methods can be used simultaneously or separately. At present, the commonly used methods for fire detection is the alpha cup cumulative radon measurement method. The detector is ionic form, which has the advantages of simple operation, light weight and high sensitivity.

3.2.2 Detection technology

a. Measuring-point arrangement. According to the spontaneous combustion situation, choose an area on the ground in advance for the arrangement of measuring points, the shape of which is square, rectangle and irregular network. In the process of measurement, the network extend in all directions based on actual situation. Dot pitch according to the requirements of the different measuring precision is $20 \mathrm{~m} \times 20 \mathrm{~m}, 10 \mathrm{~m} \times 10 \mathrm{~m}, 5 \mathrm{~m} \times 5 \mathrm{~m}$, etc. The introduction of the initial network measurement requires high precision and surface-underground contrast. 
b. Detect cup burying. Dig $30 \mathrm{~cm}$ wide, $40 \mathrm{~cm} \sim 50 \mathrm{~cm}$ deep pit in every numbered measuring point and put the detect cup in the pit upside down. Cover it with plastic cloth, bury it with earth, and at the same time record the time, periods, rock properties, etc.

c. Measurement. After probe cup was embedded within the pit for 4 h, i.e. radon decay of radioactive equilibrium state, remove probe cup and put it into instrument to measure immediately. Take a note of $1 \mathrm{~min}, 3 \mathrm{~min}$ and $5 \mathrm{~min}$ 's readings and fill in the measuring radon data card.

d. Data processing and results. Inputting the field measured data into measuring radon fire detecting data processing software (CDTH), we can get stereogram and corresponding contour map of measured value and outliers. Draw planar graph of fire range according to the results of processing. Planar graph can provide information about location, range, fire development direction of fire center, abnormal area and fire area, thus providing scientific basis for fire fighting.

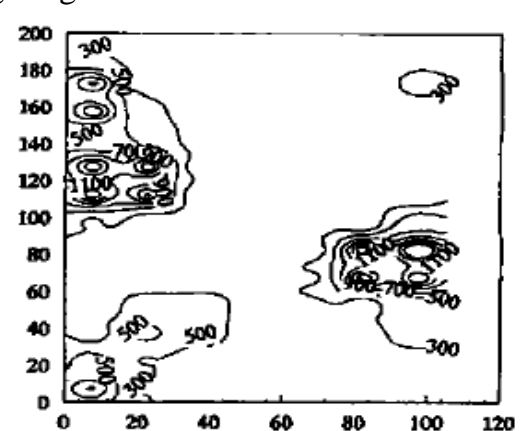

Fig 2. Measurements contour map

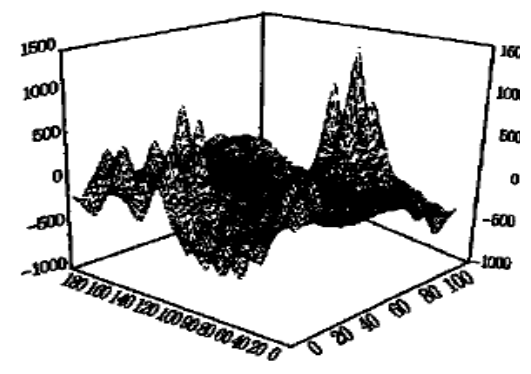

Fig 4. Outliers stereogram

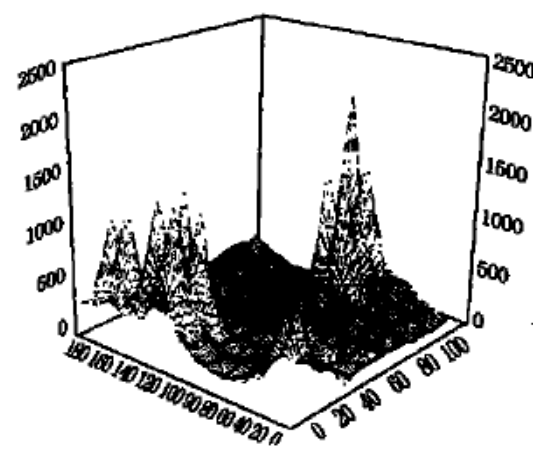

Fig 3. Measurements stereogram

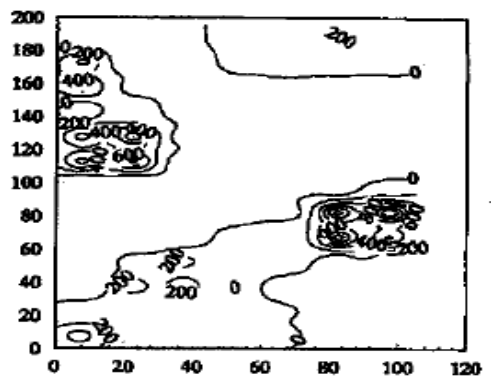

Fig 5. Outliers contour map

\section{Example analysis}

Ximing Coal Mine is part of the Western Hills Coking Coal Group. Mine area is $43.2 \mathrm{~km} 2$ and its seam dim is less than $15^{\circ}$. It has 4 minable seams, number 2 , 3, 8 and 9. Among them, NO. 8 and 9 coal are close distance coal seam, which belong to type II ignitable Coal. In April 2001, number 49309 working face of this mine occurred coal spontaneous combustion, which forced the face closed. 
The face is a compound mining face which mining overall height in one times and the mining height is 3.3 meters. The depth and the length of the face is 190 and 150meters respectively. The designed towards advance length is 750 meters.

4.1 Application of cusp catastrophe model

Through testing on site, we found the amount of $\operatorname{CO}(\Phi(\mathrm{CO}))$ in the coal spontaneous combustion period is one order of magnitude higher than the other period. Therefore, state variables $\mathrm{X}$ is chosen as $\Phi(\mathrm{CO})$. In the process of coal spontaneous combustion, the amount of carbon dioxide is selected as the major index, which reflects the internal factors influence of spontaneous combustion. Oxygen amount, coal temperature, air amount are chosen as the main index reflecting the external factors influence of coal spontaneous combustion. Relationship between Internal factor $\mathrm{u}$, external factor $\mathrm{v}$ and corresponding indexes can be respectively expressed by (10) and (11):

$$
u=a_{1} \Phi
$$

$$
\left(\mathrm{CO}_{2}\right)+\mathrm{a}_{2}
$$

$$
v=b_{1} t+b_{2} \Phi\left(O_{2}\right)+b_{3} Q+b_{4}
$$

(11)

Where, $\mathrm{t}$ is temperature, ${ }^{\circ} \mathrm{C} ; \mathrm{Q}$ is air quantity on the return air side ,m3/min; $\Phi(\mathrm{O} 2)$ is oxygen concentration ,\%; $\Phi_{(\mathrm{CO} 2)}$ is carbon dioxide concentration ,\%; a1,a2,b1,b2,b3,b4 are undetermined coefficients.

Some representative data from the data table is substituted into formula (6) ,which can be transformed into nonlinear equations after simplification:

$$
\begin{gathered}
f_{i}=\left(X_{0}-Y(C O)\right)^{3}+\left(X_{1} \Phi_{i}\left(C O_{2}\right)+\right. \\
\left.X_{2}\right)\left(X_{0}-\Phi(C O)\right)+X_{3} t+ \\
X_{4} \Phi_{i}\left(O_{2}\right)+X_{5} Q_{i}+X_{6}=0
\end{gathered}
$$

(12)

Where, X0,X1,X2,X3,X4,X5,X6 are undetermined coefficients.

Solving process of equation(13) is needed satisfies the following formula:

$$
\sum_{i=1}^{n} f^{2}=\min
$$

A set of initial values are selected to substitute into equation (13), and the coefficients are:

$\mathrm{X}_{0}=0.0174 ; \mathrm{X}_{1}=0.2456 ; \mathrm{X}_{2}=-0.0925 ; \mathrm{X}_{3}=1.1876 ; \mathrm{X}_{4}$ $=1.4832 ; X_{5}=0.00364 ; X_{7}=-61.1862$.

Therefore, coal spontaneous combustion catastrophe model of this coal mine is:

$(\mathrm{X}-0.0174)^{3}+\left(0.2456 \mathrm{Y}\left(\mathrm{CO}_{2}\right)-0.0925\right)(\mathrm{X}-0.0925)+1.1876 \mathrm{t}+1.4832$

$\mathrm{Y}\left(\mathrm{O}_{2}\right)+0.0364 \mathrm{Q}-61.1862=0$ 
Spontaneous combustion in this coal mine is analyzed, using the mutation model. Result shows that formula(14) can properly reflect the process of coal spontaneous combustion. For the samples which are closer to the right boundary of bifurcation , possibility of spontaneous combustion is greater . It should be paid enough attention in the actual production.

4.2 Application of coal seam spontaneous combustion detection technology

4.2.1 Detection field layout

According to the real condition, original detection field and boundary points are given by the Ximing Coal Mine. Its length is 150 meters and its width is 200 meters stretching to the main roadway destination. The total area of the detection field is 30 thousand $\mathrm{m} 2$. Detection field layout is shown as figure 6 .

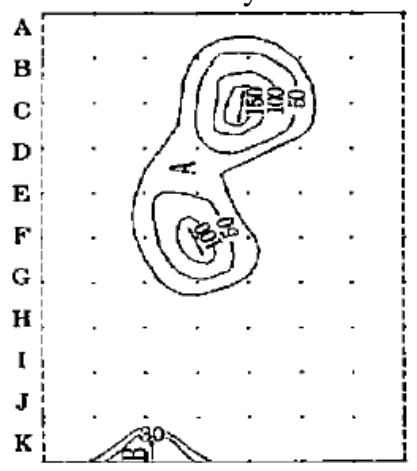

Fig.6. Fire source detection planar graph of Ximing Coal Mine

4.2.2 Analysis of detection result

Fire detection data processing software(CDTH) was used to analyze the measured data. We can get the measured value stereogram, the outlier stereogram and their corresponding isograms. Then, the fire area distribution planar graph can be got. Figure 2 and figure 3 are isograms and measured value stereograms. Figure 4 and figure 5 are the outlier stereograms and isograms, from which, the outlier area can initially be found. Combined with the actual situation, we can get figure 6 shown as Ximing Coal Mine fire source detection planar graph. As shown in figure 6, there are two high-temperature anomalous districts (A and B) and their total area is about $2580 \mathrm{~m} 2$. Two high-temperature anomalous points can be found in A district. The high temperature points are in the upper, temperature range of which is estimated from70 extension direction is northeast. High temperature points are also in the lower part, and the expected temperature range is $50{ }^{\circ} \in 150$ direction is north south direction. In addition, high temperature oxidation points can be found in B district, whose expected temperature range is $60 \quad{ }^{\circ} \in 100$

\section{Effect and conclusions}

(1)The development process of coal mine spontaneous combustion phenomena is non-linear and complex, which can be better analyzed by using catastrophe mutation theory. 
(2) Aiming at two types of dangerous sources, this article provides a new quantitative description method. Analysis of spontaneous combustion mutation in Ximing Coal Mine achieve quantitative disposal. This method can be also used to analyze and evaluate the process of other accidents .

(3) Ximing Coal Mine made a fire extinguishing plan, according to the detection result. Firstly, they drilled in the ground along the fire spread direction. Secondly, holes were arranged evenly based on long, short axis direction around the fire area, and sealed holes with grouting. In order to prevent fire area from epitaxy, the extra holes were arranged at the fire spreading area. Reinforcement and airtight measures were taken underground coal mine to eliminate the effect on other mining areas. The facts show that drilling and sealing holes in the upper part of fire area according to the detection result, not only saves a lot of money which might be used to drill the extra holes, but also improves firefighting efficiency.

\section{References}

[1] Wu Jianming, Zhai Jianshan. Study and Application of Key Technique for Extinguishing the Spontaneous Combustion in Coal Mines. China Safety Science Journal,1998(4).

[2] Fire Control Research Group of Taiyuan University of Technology. Precise Probe Technology on Coal Spantaneous Fire Location. Research Report,2000.

[3] Lin Fuhua. Catastrophe Theory and Its Application[M].Profile of Shanghai Jiao Tong University Press,1992,11

[4] Zhong Maohua, Chen Baozhi.Application of Catastrophe Theory to Mining Safety[J].China Safety Science Journal,1998,2

[5]C Marco, J Duffy, V P Castelli. Catastrophe analysis of a planar system with flexural pivots. Mechanism and Machine Theory, 2002, 37(7):693-716

[6] Tang Tieqiao Huang Haijun. The Discussion of Traffic Flow Forecast by Using Swallowtail Catastrophe Theory. Journal of Mathematical Study,2005,38(1):112-116

[7] Li Luping, HUANG Qi, Zou Xinyuan.Research on mechanism of vibration catastrophe caused by contact-rubbing in large capacity turbo-generator sets.Journal of Electric Power Science and Technology,2007,22(1):51-55

[8] Li Ming, Mao Xianbiao, Mao Rongrong, et al. Study on buckling instability of surrounding rock based on cusp catastrophe model[J]. Journal of Mining \& Safety Engineering, 2014,31(3):379-384.

[9] Lu Aihong, Zhang Lianying. Effect of the horizontal tectonic stress on floor water-inrush[J]. Journal of Mining \& Safety Engineering, 2010, 27(3): 395-398.

[10] Zhang Qinli, Cao Xiaogang, WANG Yanli, et al. Stability analysis of stope roof-pillar based on Cusp catastrophe model[J]. China Safety Science Journal, 2011, 21(10): 52-57. 
[11] Guastell S. J. Catastrophe modeling of the accident process: evaluation of an accident reduction using the occupational hazards survey[J]. Accident Anal Prev,1989.

[12] Hao Jifeng. Catastrophe Study on Mine Spontaneous Fire[D]. Taiyuan: Taiyuan University of Technology,2002.

[13] Tian Shuicheng, Li Xindong. Surroundings of Coal Spontaneous Combustion and Its Influence Factors[J].Journal of Xi'an Mining Institute,1994,2

[14] Acha-Daza, Jorge A.and Hall, Fred L.. Graphical comparison of predictions for speed given by catastrophe theory and some classic models. Transportation Research Record, 1993, 1398:119-124.

[15] Qian Xin-ming, Chen Bao-zhi. On Identification \& Control of Major Hazards. China Safety Science Journal,1994,8 